\title{
Effect of a home based, low intensity, physical exercise program in older adults dialysis patients: a secondary analysis of the EXCITE trial
}

Rossella Baggetta1', Graziella D’Arrigo', Claudia Torino', Samar Abd ElHafeez³, Fabio Manfredini², Francesca Mallamaci ${ }^{1}$, Carmine Zoccali ${ }^{1}$, Giovanni Tripepi ${ }^{*}$ and on behalf of the EXCITE Working group

\begin{abstract}
Background: Older adults dialysis patients represent the frailest subgroup of the End Stage Renal Disease (ESRD) population and physical exercise program may mitigate the age-related decline in muscle mass and function.

Methods: Dialysis patients of the EXCITE trial aged $>65$ years $(n=115$, active arm, $n=53$; control arm, $n=62$ ) were submitted in random order to a home based, low intensity physical exercise program. At baseline and 6 months after exercise training 6-min walking distance (6MWD) and 5-time sit-to-stand test (5STS) were performed, and quality of life (QoL) was tested.

Results: The training program improved both the 6MWD (6-months: $327 \pm 86 \mathrm{~m}$ versus baseline: $294 \pm 74 \mathrm{~m} ; P<0.001$ ) and the 5STS time (6-months: $19.8 \pm 5.6 \mathrm{~s}$ versus baseline: $22.5 \pm 5.1 \mathrm{~s} ; \mathrm{P}<0.001)$ in the exercise group whereas they did not change in the control group ( $P=0.98$ and 0.25 , respectively). The between-arms differences ( 6 months-baseline) in the 6MWD (+34.0 m, 95\% Cl: 14.4 to $53.5 \mathrm{~m}$ ) and in the 5STS time changes $(-1.9 \mathrm{~s}, 95 \% \mathrm{Cl}:-3.6$ to $-0.3 \mathrm{~s})$ were both statistically significant $(P=0.001$ and $P=0.024$, respectively). The cognitive function dimension of QoL significantly reduced in the control arm (P=0.04) while it remained unchanged in the active arm $(P=0.78)$ (between groups difference $P=0.05$ ). No patient died during the trial and the training program was well tolerated.
\end{abstract}

Conclusions: This secondary analysis of the EXCITE trial shows that a home-based, exercise program improves physical performance and is well tolerated in elderly ESRD patients.

Trial registration: The trial was registered in ClinicalTrials.Gov (Clinicaltrials.gov identifier: NCT01255969) on December 8 , 2010.

Keywords: Dialysis patients, Physical exercise, Quality of life

\section{Background}

Sedentary lifestyle and reduced physical performance are hallmarks of dialysis patients [1] and both these conditions negatively impact upon general health status and quality of life in this patient-population $[2,3]$. NKF-KDOQI Guidelines formally recommend patients on dialysis be encouraged to increase their level of

\footnotetext{
* Correspondence: gtripepi@ifc.cnr.it

${ }^{1}$ Clinical Epidemiology and Physiopathology of Renal Diseases and Hypertension of Reggio Calabria, National Council of Research, Institute of Clinical Physiology, Reggio Calabria, Italy

Full list of author information is available at the end of the article
}

physical activity [4] and a recent randomized, controlled, multicenter, clinical trial (Clinicaltrials.gov identifier: NCT01255969) demonstrated that a personalized, low intensity, home-based, exercise program improved physical performance and had a favourable impact on cognitive function and quality of social interaction (as assessed by KDQOL-SF) in dialysis patients [5]. Furthermore, a corollary analysis of the EXCITE trial showed that a poor physical performance, as assessed by the 6-min walking distance [6], predicted a high risk of mortality, cardiovascular events and hospitalizations in the dialysis population [7].

(c) The Author(s). 2018 Open Access This article is distributed under the terms of the Creative Commons Attribution 4.0 International License (http://creativecommons.org/licenses/by/4.0/), which permits unrestricted use, distribution, and reproduction in any medium, provided you give appropriate credit to the original author(s) and the source, provide a link to the Creative Commons license, and indicate if changes were made. The Creative Commons Public Domain Dedication waiver (http://creativecommons.org/publicdomain/zero/1.0/) applies to the data made available in this article, unless otherwise stated. 
Patients over 65 years of age constitute the fastest growing segment of ESRD population worldwide [8] and although dialysis treatment increases life expectancy among older patients on chronic dialysis, many uremic patients suffer from distressing physical limitations [9]. Since aging is associated with a decline in muscle mass and performance [10], physical exercise is one of the potential methods to counterbalance age-related worsening in muscle mass and function [11]. Notwithstanding, to date very few studies investigated the effect of physical exercise on clinical outcomes in older patients on dialysis.

With this background in mind we specifically designed a secondary analysis of the Exercise Introduction to Enhance Performance in Dialysis (EXCITE) trial [5] by investigating the efficacy and safety of a low intensity exercise program on physical performance and quality of life in the subgroup of patients of the EXCITE trial aged $>65$ years.

\section{Methods}

\section{Ethical approval and consent to participate}

The original protocol of the EXCITE trial was approved by the Ethics Committees of Renal Units $(n=9$ Italian Centers) participating to the study, and written informed consent was obtained from each participant. The Ethical Committee of the Coordinating Center is that of "Grande Ospedale Metropolitano Bianchi-Melacrino-Morelli" of Reggio Calabria, Italy (Committee's reference number: CE150157). The trial was registered in ClinicalTrials.Gov (Clinicaltrials.gov identifier: NCT01255969) on December 8, 2010.

\section{Study design}

EXCITE is a centrally randomized (simple randomization), multicenter, open label, controlled, two parallel arms, clinical trial testing the effect of a personalized, home-based, low-intensity program [see: https://www.youtube.com/ watch? $=$ ki8YX_t-0jA, 5] of walking exercise (exercise group) versus usual care (control group, receiving generic advice to maintain an active lifestyle) on physical performance [as assessed by 6-min walking distances (6MWD) and 5-time Sit-To-Stand (5STS) tests] and clinical outcomes (including quality of life) in dialysis patients [6, 12-14]. The walking cadence (steps/minute) to be maintained at home was dictated by an easily available metronome (Seiko DM50; Seiko Ltd., Japan) which was distributed to all participants. The adherence to the prescribed program was assessed by evaluating the residual battery charge in the metronome at the end of the trial. Exclusion criteria were physical or clinical limitations or a high degree of fitness, that is the ability to walk a distance of $550 \mathrm{~m}$ in $6 \mathrm{~min}$ during the standard walking test (see Ref. [6]). All eligible patients were recruited between November of 2009 and
February of 2011. This secondary analysis focuses on the subgroup of patients who took part to the EXCITE trial and aged $>65$ years $(n=115)$. The enrolment period ranged from April 2010 to February 2011.

\section{Laboratory data}

Serum cholesterol, triglycerides, hemoglobin, albumin, calcium, phosphate, PTH, glucose, and C-Reactive Protein were measured by standard methods in the routine clinical laboratory.

\section{Functional capacity tests}

The testing sessions were always arranged in a non-dialysis day, $24 \mathrm{~h}$ after the dialysis session, either in the morning (between 7 a.m. and 1 p.m.) or in the afternoon (between 2 p.m. and 6 p.m.). Functional capacity testing in both study arms (exercise and control groups) was performed at baseline and after 6 months, using the 6-MWD and the 5-STS. Fatigue during the exercise was assessed by the Borg scale. In the active arm of the trial, the exercise programme was permitted to be done at home or outdoor, dependent on patients' preferences. Low and high adherence were defined as performance of $<60 \%$ and $\geq 60 \%$ of the prescribed sessions, respectively. Patients in the control group was given just generic advice to maintain an active lifestyle.

\section{Quality of life}

Quality of life was measured by the KDQOL-SF in the version translated into Italian and specifically validated in a sample of Italian patients with CKD. Whenever needed, the compilation of the replies to the KDQOL-SF was helped by nurses unaware of the treatment allocation of patients. For the purpose of this paper, only changes occurring over a 6-month follow-up period were considered in the data analysis.

\section{Statistical analyses}

Data are expressed as mean and SD (normally distributed data), median and interquartile range (non-normally distributed data), or as percentage frequency (categorical data), and comparisons between groups were made by independent $\mathrm{t}$-test (normally distributed data), Mann-Whitney test (non-normally distributed data), or chi-squared test (categorical data), as appropriate. Within-group comparisons were done by paired $t$-test (normally distributed data) or Wilcoxon rank test (non-normally distributed data), as appropriate. Between- and within-group differences were expressed as mean changes and 95\% confidence intervals (CIs).

An intention-to-treat (ITT) approach was used for the primary study outcomes (the 6MWD and the 5STS). The potential confounding effect of baseline variables which differed (with $P<0.10$ ) between the two study 
arms on the study results was tested by multiple linear regression analysis (for changes in 6MWD and 5STS). Given the fact that this is a secondary analysis of a previous clinical trial, no sample size calculation was reported. The effect of physical exercise on the incidence rate of all-cause and cardiovascular hospitalizations according to study arms was investigated by Cox regression method. In this analysis, data were expressed as hazard ratio (HR), 95\% $\mathrm{CI}$ and $P$ value.

Data analysis was performed using a standard statistical package (SPSS for Windows, version 22; IBM SPSS, Chicago, IL, USA).

\section{Results}

The CONSORT diagram describing the flow of patients through the trial is shown in Fig. 1. The source population was composed by 714 patients and among these 418 patients were excluded for various reasons (Fig. 1). Thus, 296 patients were randomized to walking exercise $(n=151)$ or to usual care/normal physical activity $(n=$ 145). As depicted in Figs. 1, 160 patients of 296 (54\%) fulfilled the criterion to have an age over 65 years (active arm, $n=83$; control arm, $n=77$ ) and were considered in this secondary analysis. Older adults patients (i.e. patients aged $>65$ years) who completed the study at 6 months (active arm, $n=53$; control arm, $n=62$ ) in the two study arms were quite similar as for demographic, clinical, and biochemical data (Table 1) but differed for BMI, systolic BP, hemoglobin, and albumin (Additional file 1: Table S1) which were higher in patients in the active than in those in the control arm. Patients in the active arm had also less frequently stroke/TIA in their clinical history (Table 1). Moreover, age tended to be lower and diastolic $\mathrm{BP}$ to be higher $(P=0.06$ and $P=0.07$, respectively) in patients allocated to the active arm than in those to the control arm (Table 1). Among patients allocated to the active arm, 26 had low (49\%) and 27 high (51\%) adherence to the physical exercise program.

\section{Effect of the home-based training program on functional capacity and other parameters}

At Baseline, 6MWD and 5STS time did not significantly differ between the two study groups (Table 1). At 6 months, both the 6MWD (6 months: $327 \pm 86 \mathrm{~m}$ versus baseline: $294 \pm 74 \mathrm{~m}$; within-group comparison, $P<0.001)$ and the 5STS time (6 months: $19.8 \pm 5.6 \mathrm{~s}$ versus baseline: $22.5 \pm$ $5.1 \mathrm{~s}$; within-group comparisons, $P<0.001)$ improved in the exercise group whereas they did not change in the control group (6MWD, 6 months: $270 \pm 98$ m versus baseline: $271 \pm 92 \mathrm{~m}$; within-group comparison, $P=0.98$; 5STS, 6 months: $23.1 \pm 5.8 \mathrm{~s}$ versus $23.9 \pm 5.3 \mathrm{~s}$; within-group comparison, $P=0.25$ ) (Table 2). The between-arms differences (6 months-baseline) in the 6MWD (+ $34.0 \mathrm{~m}, 95 \% \mathrm{CI}: 14.4$ to $53.5 \mathrm{~m})$ and in the 5STS time changes $(-1.9 \mathrm{~s}, 95 \% \mathrm{CI}:-3.6$ to $-0.3 \mathrm{~s})$ were both statistically significant $(P=0.001$ and $P=0.024$, respectively) (Table 2 ). The between-arms difference in 6MWD and 5STS time changes remained significant $(P<0.001$ and $P=0.034$, respectively $)$ also after

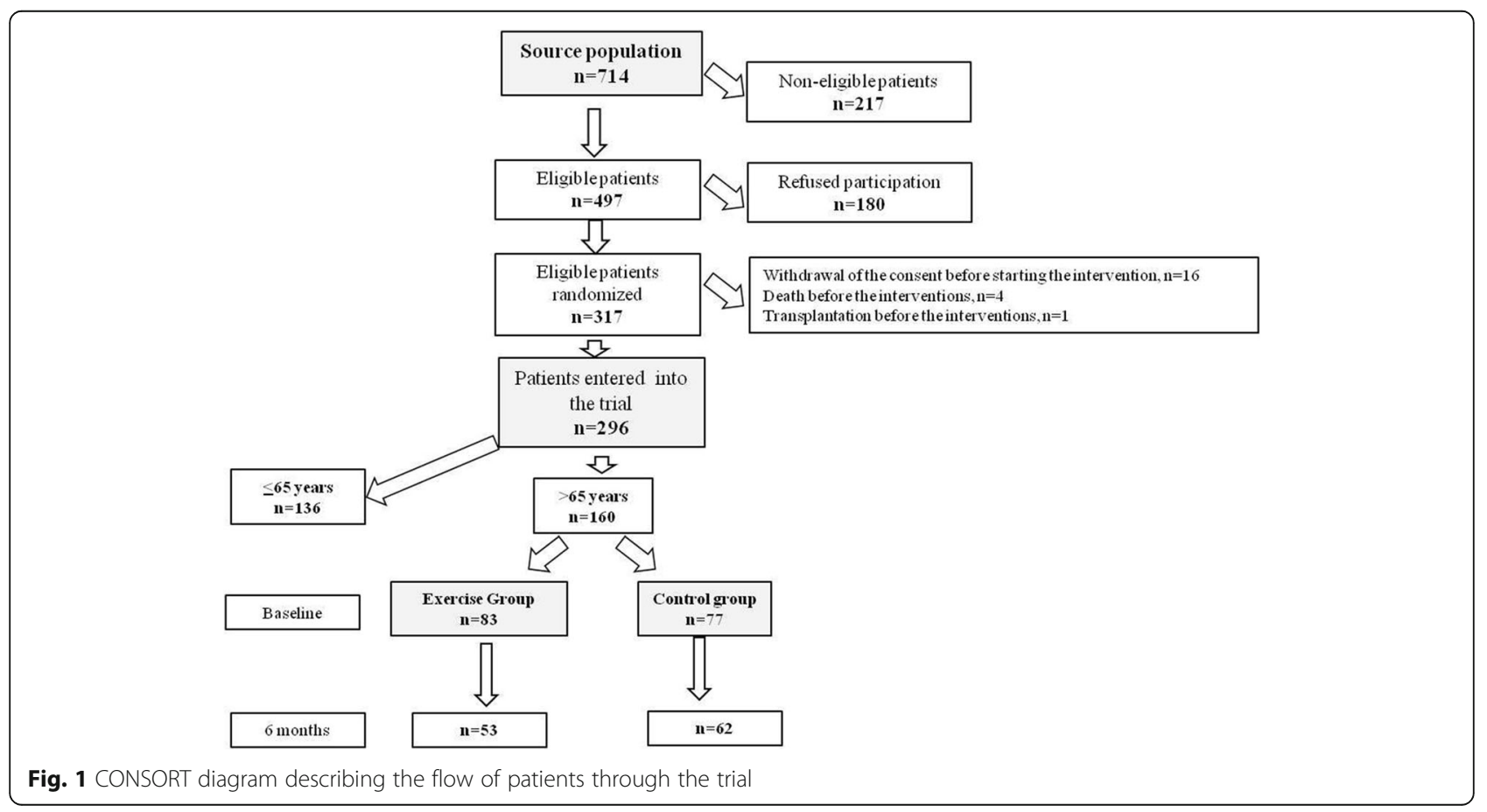


Table 1 Demographic, clinical and biochemical data of patients that completed the study

\begin{tabular}{|c|c|c|c|}
\hline & $\begin{array}{l}\text { Active arm } \\
(n=53)\end{array}$ & $\begin{array}{l}\text { Control arm } \\
(n=62)\end{array}$ & P Value \\
\hline Age, yr & $73 \pm 5$ & $75 \pm 6$ & 0.06 \\
\hline Men, \% & 64 & 66 & 0.82 \\
\hline Hemodialisys/CAPD, n & $44 / 9$ & $55 / 6$ & 0.25 \\
\hline $\mathrm{BMl}, \mathrm{Kg} / \mathrm{m} 2$ & $27 \pm 4$ & $25 \pm 3$ & 0.01 \\
\hline Smokers, \% & 17 & 8 & 0.16 \\
\hline Diabetics, \% & 17 & 20 & 0.75 \\
\hline Myocardial infarction, \% & 15 & 19 & 0.55 \\
\hline $\begin{array}{l}\text { Stroke/transient ischemic } \\
\text { attack, \% }\end{array}$ & 4 & 16 & 0.03 \\
\hline Anginal episodes, \% & 11 & 16 & 0.46 \\
\hline Arrhythmia, \% & 17 & 13 & 0.54 \\
\hline Heart failure, \% & 19 & 27 & 0.28 \\
\hline Peripheral vascular disease, \% & 7 & 16 & 0.16 \\
\hline History of neoplasia, \% & 21 & 28 & 0.38 \\
\hline Antihypertensive therapy, \% & 78 & 64 & 0.11 \\
\hline NYHA class, \% & & & 0.32 \\
\hline । & 40 & 33 & \\
\hline$\|$ & 36 & 37 & \\
\hline III-IV & 23 & 30 & \\
\hline \multicolumn{4}{|l|}{ Mobility, \% } \\
\hline Assisted & 6 & 5 & 0.86 \\
\hline Independent & 94 & 95 & \\
\hline $\begin{array}{l}\text { Six-Minute Walking } \\
\text { Distance, mts }\end{array}$ & $294 \pm 74$ & $271 \pm 92$ & 0.14 \\
\hline $\begin{array}{l}\text { 5-time Sit-to Stand } \\
\text { Test, sec }\end{array}$ & $22.5 \pm 5.1$ & $23.9 \pm 5.3$ & 0.16 \\
\hline
\end{tabular}

data adjustment for baseline variables which differed (with $P<0.10$ ) between the two study arms as well as for baseline 6MWD and 5STS time.

No significant effect of physical exercise was found on the incidence rate of all-cause [HR (active versus control arm): $0.68,95 \%$ CI: $0.25-1.87, P=0.46]$ and cardiovascular hospitalizations [HR: 1.18, 95\% CI: $0.38-3.66, P=0.78$ ].

Changes in clinical and biochemical biomarkers occurring over the 6-month period are given in Additional file 1: Table S1.

\section{Quality of life}

Within and between-arms differences of changes in KDQOL-SF components occurring over the 6-month period are detailed in Additional file 1: Table S2. As shown in Additional file 1: Table S2, cognitive function significantly reduced in patients of the control arm $(P=0.04)$ while it remained substantially unchanged in those of the active arm $(\mathrm{P}=0.78)$ and the between-arms difference was statistically significant $(P=0.05)$. No other statistically significant differences were found between-groups differences in changes of KDQOL-SF components (Additional file 1: Table S2).

\section{Safety of the exercise program}

No patient died during the trial. No angina episode or other major symptoms/complications during exercise were reported in the active arm of the trial. Symptoms of moderate intensity, not limiting the program execution, were reported in $54 \%$ of the session performed by 38 patients and included moderate fatigue $(n=31)$, "heavy legs" or leg pain $(n=29)$, moderate dyspnoea $(n$ $=19)$, or other symptoms, including joint pain $(n=14)$. Six patients reported four symptoms, 12 reported three symptoms, 14 reported at least two symptoms, and 6 only reported one symptom during the exercise sessions. Overall, the training program was well tolerated and only 5 telephone calls were received by the rehabilitation team across the trial.

\section{Discussion}

This secondary analysis of the EXCITE trial generates the hypothesis that a low intensity, home-based, personalized, physical exercise program improves physical performance and stabilizes cognitive function in dialysis patients aged $>65$ years.

Dialysis patients have extremely low levels of physical functioning and exercise capacity, and are often physically inactive $[15,16]$. NKF-KDOQI Guidelines formally recommend that all dialysis patients should be counselled and regularly encouraged by nephrology and dialysis staff to increase their level of physical activity and that physical functioning assessment and encouragement for participation in physical activity should be part of the routine patient care plan [4]. On the basis of this recommendation, we designed and performed a multicenter,

Table 2 Within and between-arms differences in 6MWD and 5STS time. Data are mean and standard deviation or as mean and 95\% $\mathrm{Cl}$

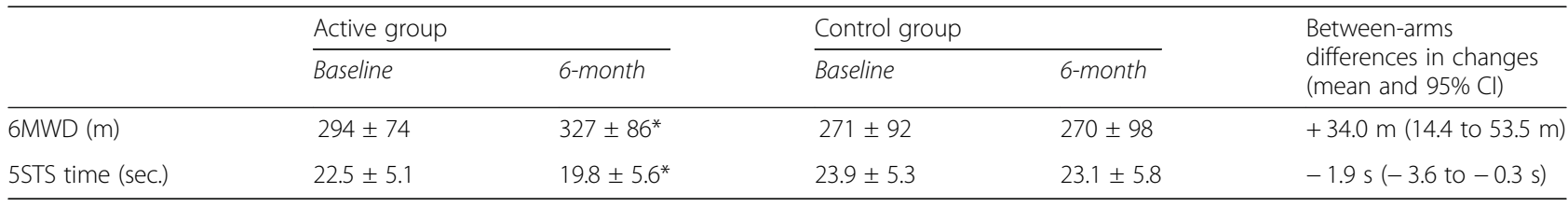

${ }^{*} P<0.001$ versus baseline 
randomized, controlled clinical trial, the EXerCise Introduction To Enhance performance in dialysis patients trial (EXCITE -NCT01255969), testing whether a personalized, home-based, low-intensity, physical exercise program improves the degree of fitness and quality of life in dialysis patients $[5,7,17]$. In the primary analysis of the EXCITE trial, i.e. in a dialysis population with a wide spectrum of age (from 25 to 91 years), we already documented that a home-based exercise program managed by dialysis staff improves physical performance and quality of life in dialysis patients [5]. In particular, over a 6 -month time period we found that the 6MWD improved by $39 \mathrm{~m}$ in the exercise group and only by $3 \mathrm{~m}$ in the control group and the between groups difference was highly significant $(P<0.001)$. Similarly, the 5STS test time improved in the active arm $(-2.3 \mathrm{~s})$ whereas it remained virtually unchanged in the control arm of the same trial $(-0.7 \mathrm{~s})$ and again the between groups difference was statistically significant $(P=0.001)$. In the present analysis restricted to ESRD patients aged > 65 years, we found that in patients of the active arm the improvements in 6MWD $(+33 \mathrm{~m})$ and in 5STS test time $(-2.7 \mathrm{~s})$ were of similar magnitude to those found in patients of the exercise arm of the primary analysis of the EXCITE trial $(\Delta$ 6MWD: $+39 \mathrm{~m} ; \Delta$ 5STS: $-2.3 \mathrm{~s})$ whereas no improvement in physical performance was observed in older adults patients of the control arm $(\Delta$ 6MWD: $-1 \mathrm{~m}, \Delta 5 \mathrm{STS}$ test time: $-0.8 \mathrm{~s}$ ). These findings are of interest because for the first time we demonstrate that a low intensity, home-based, physical exercise program has an effect in older adults dialysis patients which is comparable to that observed in the general ESRD population with a wide age range (from 25 to 91 years).

The EXCITE trial also contemplated the assessment of quality of life in patients of the active and control arm over a 6-month time period. Cognitive function is an important component of quality of life and general health status which pertains attention, memory, language, perception, decision making, and problem solving [18-20]. The burden of cognitive impairment is higher in dialysis patients than in the general population and represents a disabling condition because it compromises quality of life, increases resource utilization, and results in suboptimal medical care [21-23]. In this secondary analysis of the EXCITE trial, we found that cognitive function significantly reduced in patients of the control arm $(P=0.04)$ while it remained substantially unchanged in those of the active arm $(P=0.78)$ and the between-arms difference was of borderline significance $(P=0.05)$. This result suggests that regular physical activity over a 6 -month time period may stabilize the cognitive decline in older adults dialysis patients and generates the hypothesis that physical exercise may counterbalance the age-related worsening in cognitive function in the dialysis population. However, this latter hypothesis demands to be specifically investigated in an appropriately designed randomized clinical trial.

The effect of physical exercise on clinical outcomes in dialysis patients represents a tantalizing research area and various exercise programmes have been proposed. Koh et al., in a pilot study in older dialysis patients [24], found no significant differences between intradialytic or home-based exercise training and usual care for physical function as assessed by 6MWD. The results emerged in our study do not contrast with those of Koh et al. In the Koh's study, over a 6 month period, the increase in 6MWD in patients of the home-based exercise arm was more than double than that found in patients of the usual care arm (11\% versus $5 \%)$ and the lack of a significant difference between the magnitude of these effects most likely depends on the extremely low sample size of the Koh's study (from an initial population of 70 patients, the 6 month data were only available in 15 patients of the home-based exercise arm and in 16 patients of the usual care arm).

Another novel finding of our study is that the physical exercise program is safe and well tolerated in older adults ESRD patients. Moderate intensity symptoms such as moderate fatigue, leg pain, dyspnea and joint pain were recorded in $54 \%$ of session performed by 38 patients and only 5 phone calls for problems related with exercise tolerance were received by the rehabilitation team that supervised the training of patients and the safety of the intervention. Furthermore, no episodes of angina or other major symptoms have been reported in either of the two analyzed groups.

Major limitations of our study are that it is a post-hoc analysis of a randomized clinical trial which did not aim to examine a geriatric population and the fact that the number of patients considered here is about one half of that of the original trial (115 versus 227). Thus, our findings need to be interpreted very cautiously and formally confirmed in a randomized clinical trial specifically enrolling older adult patients on dialysis. Another limitation of our study is that the protocol did not contemplate frailty assessment, dietary change or dietary diary and other physical assessment.

\section{Conclusions}

In conclusion, this secondary analysis of the EXCITE trial generates the hypothesis that a simple, home-based, personalized exercise program is well tolerated and improves physical performance and stabilizes cognitive function in older adults dialysis patients. Our trial represents a stimulus to nephrologists for beginning long term trials testing whether a simple exercise training could reduce the risk of many adverse health conditions 
and increase life expectancy and quality of life in a very high-risk population such as older adults patients on chronic dialysis.

\section{Additional file}

Additional file 1: Table S1. Within and between arms differences in hemodynamic and biochemical data. Table S2. Within and between arms differences in KDQOL-SF components (DOC $78 \mathrm{~kb}$ )

\section{Abbreviations}

5STS: 5 Sit-To-Stand; 6MWD: 6 Minute Walking Distance; BMI: Body Mass Index; BP: Blood Pressure; CKD: Chronic Kidney Disease; ESRD: End-Stage Renal Disease; EXCITE: EXerCise Introduction To Enhance; ITT: Intention To Treat; KDQOL-SF: Kidney Disease Quality of Life- Short Form; NKFKDOQI: National Kidney Foundation-Kidney Disease Outcomes Quality Initiative; PTH: Parathyroid Hormone; QoL: Quality of Life; SD: Standard Deviation; TIA: Transient Ischemic Attack

\section{Acknowledgements}

Collaborators (EXCITE Working Group):

Davide Bolignanoł, Nicola Lamberti*, Silvio Bertolill, Daniele Ciurlinoll, Lisa Rocca-Reyll, Antonio Barillàף, Yuri Battagliaף, Renato Mario Rapanà**, Alessandro Zuccalä**, Graziella Bonannot†, Pasquale Fatuzzołł, Francesco Rapi-

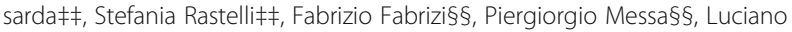
De Paolalll, Luigi Lombardilll,, Adamasco Cupistiף tano Lucisano***, Chiara Summaria***, Michele Felisatti*, Enrico Pozzato*, Anna Maria Malagoni*, Pietro Castellinołキ, Filippo Aucellat††, Pasquale Fabio Provenzanoł, Luigi Catizoneq.

IINephrology and Dialysis Unit, IRCCS Multimedica-Policlinico Multispecialistico, Milan, Italy;

INephrology and Dialysis Unit, St. Anna University Hospital, Ferrara, Italy; **Nephrology and Dialysis Unit, Ospedale Civile, Imola, Italy;

t†Azzurra Medical Nephrological Ambulatory and Dialysis Techniques, Catania, Italy;

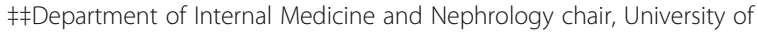
Catania, Catania, Italy;

$\S \S N e p h r o l o g y$ and Dialysis Unit, IRCCS Cà Granda Ospedale MaggiorePoliclinico, Milano, Italy;

IIIINephrology and Dialysis Unit, Ospedale Pugliese-Ciaccio, Catanzaro, Italy: 99 Department of Clinical and Experimental Medicine, University of Pisa, Pisa, Italy;

***Nephrology and Dialysis Unit, Policlinico Universitario Mater Domini, Catanzaro, Italy;

†††Nephrology Unit, Ospedale Casa Sollievo della Sofferenza, San Giovanni Rotondo, Foggia, Italy; and.

\section{Funding}

The study was supported by a Grant [Dipartimento Tutela della Salute Politiche Sanitarie - prot. 5026 del 17 febbraio 2009] of the Italian Minister of Health and by National Research Council of Italy.

\section{Availability of data and materials}

The dataset used and/or analyzed during the current study available from the corresponding author on reasonable request.

\section{Authors' contributions}

RB and GT: conceived the study, performed statistical data analysis and wrote the paper. GD, CT and SAE: were responsible of the database and performed statistical data analysis with $R B$. and $G T$. DB, NL, SB, DC, L R-R, AB, $Y B, R M R, A Z, G B, P F, F R, S R$, FF, PM, LDP, LL, AC, GF, GL, CS, MF, EP, AMM, PC, FA, PFP, and LC: coordinated the study at centre level and performed data collection and patients management. FM1, FM2 and CZ: conceived the EXCITE trial and critically revised the paper giving an important intellectual contribution. All authors read and approved the final manuscript.

\section{Ethics approval and consent to participate}

The original protocol of the EXCITE trial was approved by the Ethics Committees of Renal Units participating to the study, and written informed consent was obtained from each participant. The Ethical Committee of the Coordinating Center is that of "Grande Ospedale Metropolitano Bianchi-Melacrino-Morelli" of Reggio Calabria, Italy (Committee's reference number: CE150157). The trial was registered in ClinicalTrials.Gov (Clinicaltrials.gov identifier: NCT01255969) on December 8, 2010.

\section{Consent for publication}

Not applicable

\section{Competing interests}

The authors declare that they have no competing interests.

\section{Publisher's Note}

Springer Nature remains neutral with regard to jurisdictional claims in published maps and institutional affiliations.

\section{Author details}

${ }^{1}$ Clinical Epidemiology and Physiopathology of Renal Diseases and Hypertension of Reggio Calabria, National Council of Research, Institute of Clinical Physiology, Reggio Calabria, Italy. ${ }^{2}$ Department of Biomedical and Surgical Specialties Sciences, Section of Sport Sciences and Vascular Diseases Center, University of Ferrara, Ferrara, Italy. ${ }^{3}$ Epidemiology Department, High Institute of Public Health-Alexandria University, Alexandria, Egypt.

Received: 17 October 2017 Accepted: 8 October 2018

Published online: 20 October 2018

\section{References}

1. Johansen KL, Chertow GM, Ng AV, Mulligan K, Carey S, Schoenfeld PY, KentBraun JA. Physical activity levels in patients on hemodialysis and healthy sedentary controls. Kidney Int. 2000;57:2564-70.

2. Painter P. Implementing exercise: what do we know? Where do we go? Adv Chronic Kidney Dis. 2000;16:536-44.

3. Manfredini F, Lamberti N, Malagoni AM, Felisatti M, Zuccalà A, Torino C, Tripepi G, Catizone L, Mallamaci F, Zoccali C. The role of deconditioning in the end-stage renal disease myopathy: physical exercise improves altered resting muscle oxygen consumption. Am J Nephrol. 2015;41:329-36.

4. KDOQI. Clinical practice guidelines for cardiovascular disease in dialysis patients. Am J Kidney Dis. 2005:45:16-153.

5. Manfredini F, Mallamaci F, D'Arrigo G, Baggetta R, Bolignano D, Torino C, Lamberti N, Bertoli S, Ciurlino D, Rocca-Rey L, Barillà A, Battaglia Y, Rapanà RM, Zuccalà A, Bonanno G, Fatuzzo P, Rapisarda F, Rastelli S, Fabrizi F, Messa P, De Paola L, Lombardi L, Cupisti A, Fuiano G, Lucisano G, Summaria C, Felisatti M, Pozzato E, Malagoni AM, Castellino P, Aucella F, Abd ElHafeez S, Provenzano PF, Tripepi G, Catizone L, Zoccali C. Exercise in patients on dialysis: a multicenter, randomized clinical trial. J Am Soc Nephrol. 2017;28:1259-68.

6. ATS Committee on proficiency standards for clinical pulmonary function laboratories. ATS statement: guidelines for the six-minute walk test. Am J Respir Crit Care Med. 2002;166:111-7.

7. Torino C, Manfredini F, Bolignano D, Aucella F, Baggetta R, Barillà A, Battaglia Y, Bertoli S, Bonanno G, Castellino P, Ciurlino D, Cupisti A, D’Arrigo G, De Paola L, Fabrizi F, Fatuzzo P, Fuiano G, Lombardi L, Lucisano G, Messa P, Rapanà R, Rapisarda F, Rastelli S, Rocca-Rey L, Summaria C, Zuccalà A, Tripepi G, Catizone L, Zoccali C, Mallamaci F. EXCITE working group. Physical performance and clinical outcomes in dialysis patients: a secondary analysis of the EXCITE trial. Kidney Blood Press Res. 2014;39:205-11.

8. Singh P, Germain MJ, Cohen L, Unruh M. The elderly patient on dialysis: geriatric considerations. Nephrol Dial Transplant. 2014;29:990-6.

9. Berger JR, Hedayat SS. Renal replacement therapy in the elderly population. Clin J Am Soc Nephrol. 2012;7:1039-46.

10. Keller $\mathrm{K}$, Engelhardt M. Strength and muscle mass loss with aging process. Age and strength loss. Muscles Ligaments Tendons J. 2013;3(4):346-50.

11. World Health Organisation. Global recommendations on physical activity for health. Geneva: World Health Organisation; 2010.

12. Mong Y, Teo TW, Ng SS. 5-repetition sit-to-stand test in subjects with chronic stroke: reliability and validity. Arch Phys Med Rehabil. 2010;91:407-13.

13. Klersy C, Callegari A, Giorgi I, Sepe V, Efficace E, Politi P. Pavia working group on QoL in organ transplant. Italian translation, cultural adaptation and validation of KDQOL-SF, version 1.3, in patients with severe renal failure. J Nephrol. 2007;20(1):43-51. 
14. Hays RD, Kallich JD, Mapes DL, Coons SJ, Carter WB. Development of the kidney disease quality of life (KDQOL) instrument. Qual Life Res. 1994;3(5):329-38.

15. Kopple JD, Storer T, Casburi R. Impaired exercise capacity and exercise training in maintenance hemodialysis patients. J Ren Nutr. 2005;15(1):44-8.

16. Sun F, Norman IJ, While AE. Physical activity in older people: a systematic review. BMC Public Health. 2013;13:449.

17. Baggetta R, Bolignano D, Torino C, Manfredini F, Aucella F, Barillà A, Battaglia Y, Bertoli S, Bonanno G, Castellino P, Ciurlino D, Cupisti A, D'Arrigo G, De Paola L, Fabrizi F, Fatuzzo P, Fuiano G, Lombardi L, Lucisano G, Messa P, Rapanà R, Rapisarda F, Rastelli S, Rocca-Rey L, Summaria C, Zuccalà A, Abd ElHafeez S, Tripepi G, Catizone L, Mallamaci F, Zoccali C. EXCITE working group. Fitness for entering a simple exercise program and mortality: a study corollary to the exercise introduction to enhance performance in dialysis (EXCITE) trial. Kidney Blood Press Res. 2014;39(2-3):197-204.

18. First MB, editor. American Psychiatric Association diagnostic and statistical manual. 4th ed. Washington DC: APA Press; 1994.

19. Knopman DS, Boeve BF, Petersen RC. Essentials of the proper diagnoses of mild cognitive impairment, dementia, and major subtypes of dementia. Mayo Clin Proc. 2003;78:1290-308.

20. Petersen RC, Doody R, Kurz A, Mohs RC, Morris JC, Rabins PV, Ritchie K, Rossor M, Thal L, Winblad B. Current concepts in mild cognitive impairment. Arch Neurol. 2001:58:1985-92.

21. Pereira AA, Weiner DE, Scott T, Sarnak MJ. Cognitive function in dialysis patients. Am J Kidney Dis. 2005;45:448-62.

22. Sehgal AR, Grey SF, DeOreo PB, Whitehouse PJ. Prevalence, recognition, and implications of mental impairment among hemodialysis patients. Am J Kidney Dis. 1997;30:41-9.

23. Fazekas G, Fazekas F, Schmidt R, Kapeller P, Offenbacher H, Krejs GJ. Brain MRI findings and cognitive impairment in patients undergoing chronic hemodialysis treatment. J Neurol Sci. 1995;134:83-8.

24. Koh KP, Fassett RG, Sharman JE, Coombes JS, Williams AD. Effect of intradialytic versus home-based aerobic exercise training on physical function and vascular parameters in hemodialysis patients: a randomized pilot study. Am J Kidney Dis. 2010;55:88-99.

Ready to submit your research? Choose BMC and benefit from:

- fast, convenient online submission

- thorough peer review by experienced researchers in your field

- rapid publication on acceptance

- support for research data, including large and complex data types

- gold Open Access which fosters wider collaboration and increased citations

- maximum visibility for your research: over $100 \mathrm{M}$ website views per year

At $\mathrm{BMC}$, research is always in progress.

Learn more biomedcentral.com/submissions 